\title{
A CASE OF INFANTILE CORTICAL HYPEROSTOSIS
}

\author{
By Patrick J. Sweeney, M.D., F.R.C.P.I., M.R.C.P. \\ Consultant Physician, Fermanagh County Hospital, Enniskillen
}

Infantile cortical hyperostosis was first described by Caffey in 1945. Though the disease has been frequently recorded in the U.S.A., only nine cases have been recorded in British literature. The fcllowing case report is therefore of interest.

A female child, E.R., aged three months, was

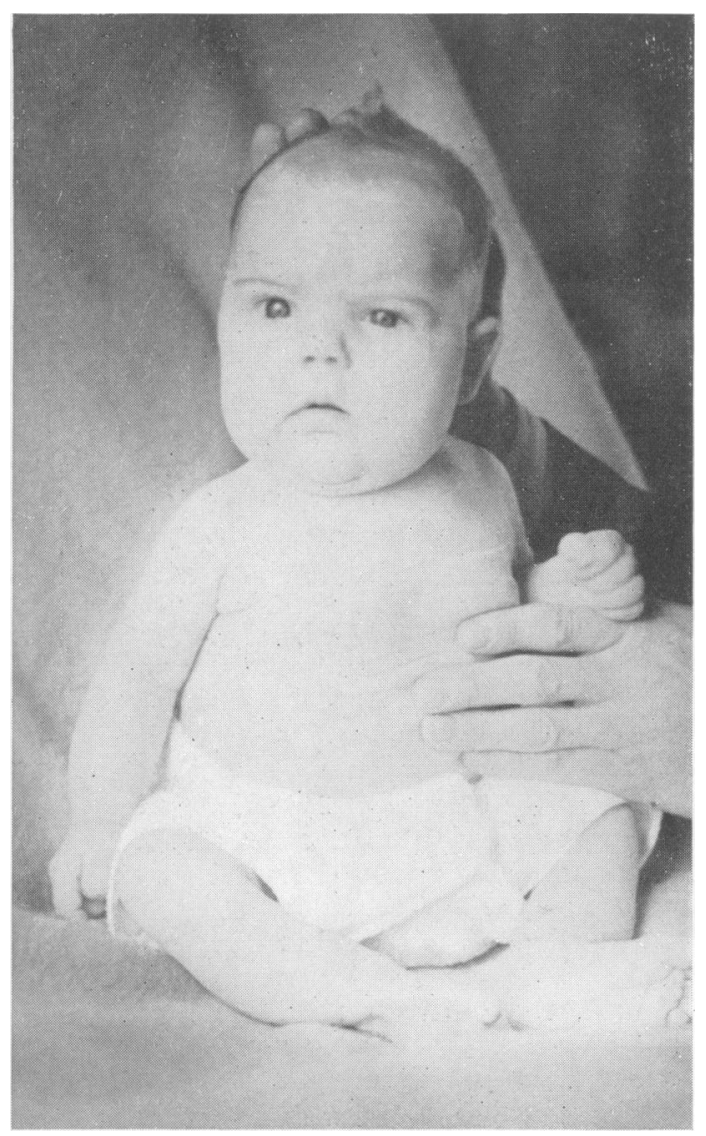

FIG. I.-Typical facies due to thickening of the mandible. admitted to hospital on March 26, 1958. For two 응 weeks prior to admission the child had been 3 irritable, with low-grade pyrexia for which no cause could be found.

Clinical examination revealed no abnormality, but it was thought that the child's appearance was $i r$ rather unusual. The cheeks and the jaw were $\bigodot_{\infty}$ excessively pudgy, and though it was not appre- $\frac{0}{3}$ ciated at that time, the facies was quite typical of infantile cortical hyperostosis (Fig. I). During $z$ the course of the next two weeks, the temperature varied between $99^{\circ}$ and $102^{\circ}$, but never became $\frac{\Phi}{3}$ normal. A chest X-ray on March 27, 1958, re vealed no abnormality; a blood count revealed considerable leucocytosis-I9,400 W.B.C., which 57 per cent. were polymorphs and $4 \mathrm{I}$ per. cent. lymphocytes. A moderately severe hyp $\overline{2}$

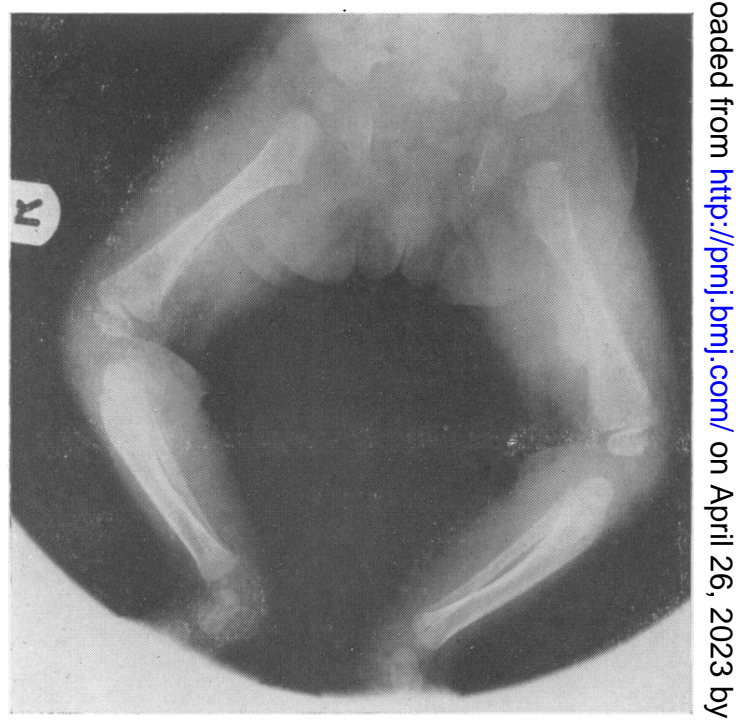

FIG. 2.-Early stage of infantile cortical hyperostosis showing new bone formation on the middle of the shaft of the left femur and early periosteal thicken-? ing of the right femur. 
chromic anaemia was present-HB. 57 per cent. (Haldane). The C.S.F. was normal.

The possibility of achondroplasia was considered and on April 9, 1958, the lower limbs were $\mathrm{X}$-rayed. The periosteal calcification on the middle of the shaft of the left femur gave the clue

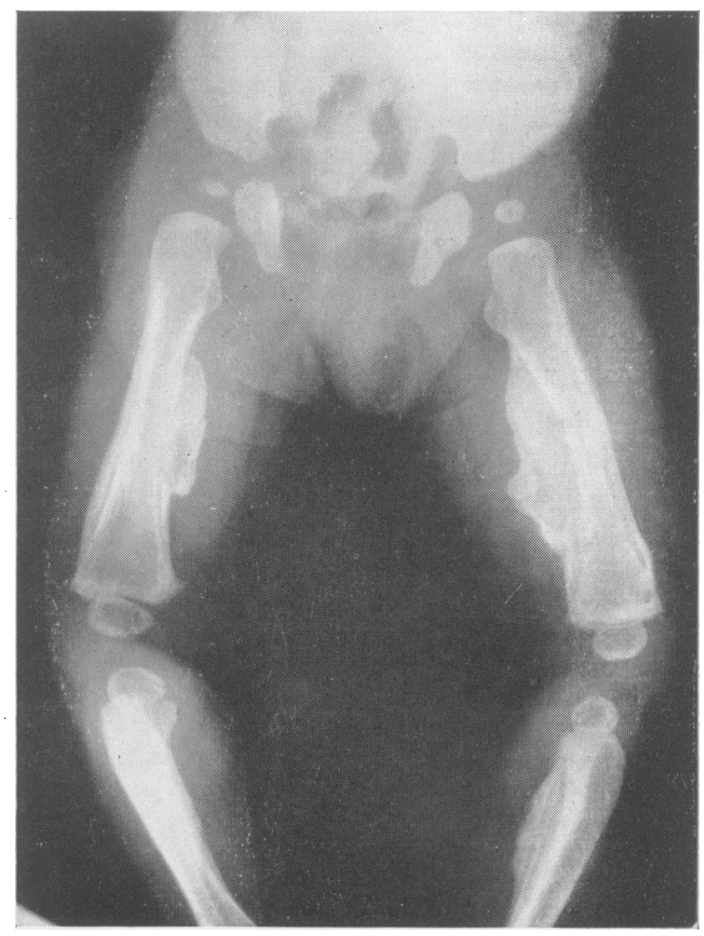

FIG. 3.-X-ray 24/4/58. Compare with Fig. 2.

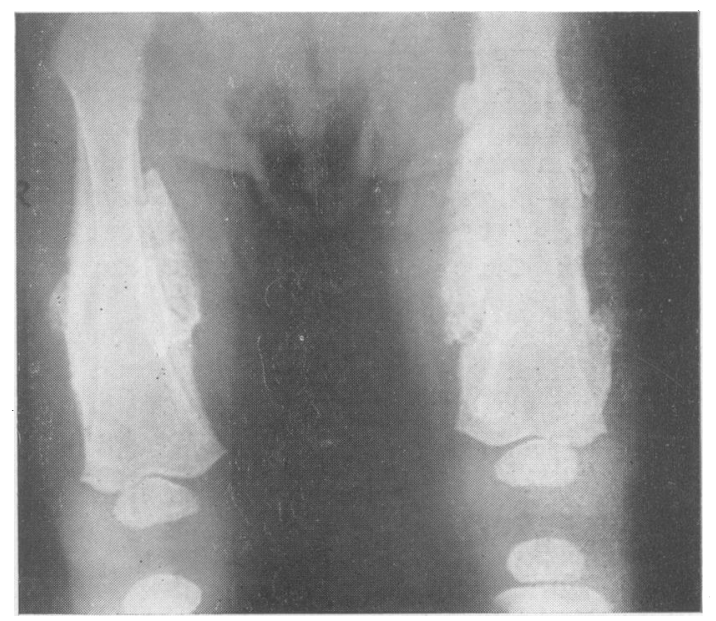

FIG. 4.- Showing extent of progression of disease in comparison to Fig. 2 of $9 / 4 / 58$. to the diagnosis (see Fig. 2), and subsequent $\mathrm{X}$-rays amply confirmed the presence of severe cortical hyperostosis.

\section{Discussion}

Caffey states that prior to 1930 there were no recorded descriptions of any similar syndrome. The disease is one of irregular new bone formation affecting long and flat bones and no valid case has ever been described where the age of onset was later than five months. It has been recognized radiologically in the 3 Ist week of pregnancy (Bennett and Nelson, 1953).

It may present as an irritable feverish child, as in the present case, or a palpable swelling may first bring it to notice (Golden, 1955). The bones

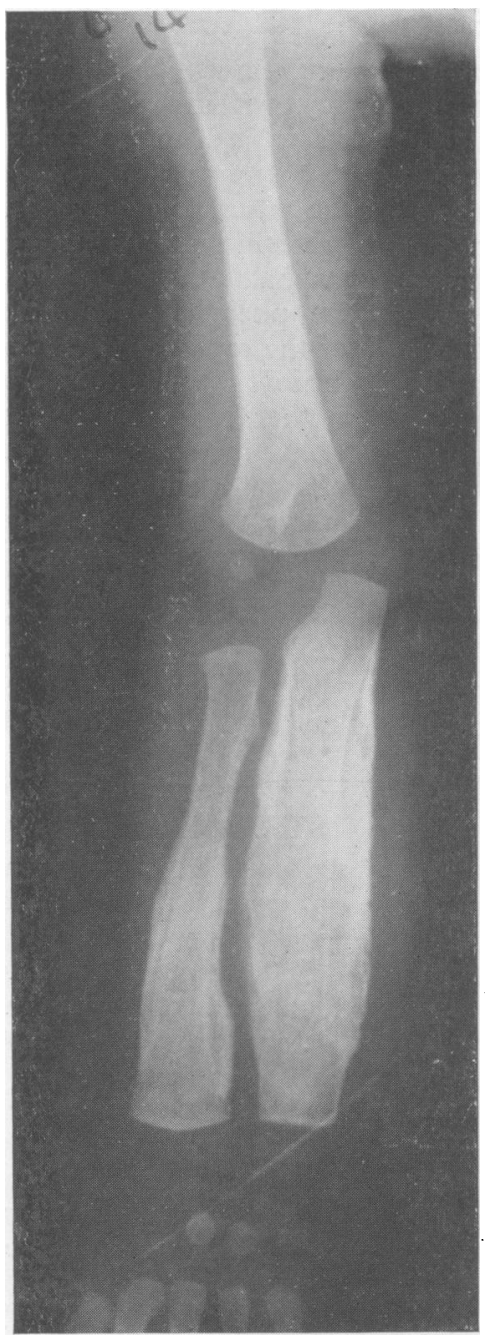

FIG. 5.-Massive deposition of new bone on the shafts of the radius and ulna. 
most commonly affected are the mandible, clavicles and ribs. Visible swellings, e.g., of a clavicle, have been known to persist for up to 20 months without regression, and facial swelling and fever have recurred for as long as seven years. On the other hand, in mild cases resolution may occur in six to eight months (Curtin, I952; Golden, 1955). The clinical course is, therefore, variable, but what is most remarkable is the speed at which new bone formation occurs. This is well shown in Figs. 3 and 4. Involvement of the radius and ulna is shown in Fig. 5, and thickening of the mandible was also present.

The radiological changes are so distinct in this disease that a differential diagnosis rarely arises. Hypervitaminosis A, which perhaps most closely simulates cortical hyperostosis, only occurs in children who are more than a year old (Snapper, 1957), and scurvy, rickets, congenital syphilis and osteomyelitis scarcely merit mention. The etiology is unknown, all serological and biochemical investigations to date having proved unhelpful. In the present case, the serum calcium was $10.4 \mathrm{mg}$. per cent., serum phosphorus 5.6 per cent., and serum alkaline phosphatase 39 units per $100 \mathrm{ml}$. The raised alkaline phosphatase is only to be expected in view of the rapid bone formation.

Though Caffey believes that the etiology should be sought in the mother, rather than in the child, questioning the mother in the present case failed to extract any useful information. She has one other child, a boy of five, who has always been well. Before and during her recent pregnancy she was in good health. She took no drugs of any kind. Delivery was normal.

While the prognosis is almost uniformly good, $\stackrel{\odot}{\circ}$ the constitutional upset can be severe, and cortisone has been found useful in shortening the course of the disease. Prednisone was given with apparent good effect in the present case-starting with $2.5 \mathrm{mg}$. four times daily-and reducing slowly to a maintenance dose of $2.5 \mathrm{mg}$. twice daily.

The temperature became normal, irritability and fretfulness disappeared, a steady gain in weight took place, and the anaemia responded satisfactorily to iron by mouth.

The child was discharged from hospital on June 12, 1958. In view of the obvious dangers, it is not proposed to take more than an X-ray of one femur every three months in following the expected regressive changes.

\section{Acknowledgments}

My thanks are due to the Photographic Deaartment of the Royal Victoria Hospital, Belfast, for photographing the $\mathrm{X}$-rays.

\section{BIBLIOGRAPHY}

BENNETT, H. S., and NELSON, T. R. (1953), Brit. F. Radiob 26, 47.

CAFFEY, J., and SILVEMAN, W. A. (1945), Amer. F. Roentgenof 54, $\mathrm{I}$.

CAFFEY, J. (1957), Proc. roy. Soc. Med., 50, 347

CURTIN, M. (1952), Irish f. med. Sci., 319, 329.

GOLDEN, G. H. (1955), Proc. roy. Soc. Med., 48, 162.

MATHEWSEN, W. J., and MARKHAM, M. (r952), Brit. med. $\mathcal{F}$. i, 747 .

SNAPPER, I. (I957), 'Bone Diseases in Medical Practice.'

\section{Mlanufacturers}

,

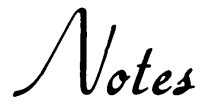

\section{STREPSILS}

Boots Pure Drug Company Limited announce the introduction of Strepsils. Strepsils are a new type of lozenge containing Dybenal $(2: 4$ dichlorobenzyl alcohol), a new antiseptic developed in the research laboratories of Boots Pure Drug Company. Laboratory tests have shown that Strepsils are lethal to many bacteria in one minute: Strepsils are effective against a wide range of bacteria and fungi. The indications include most common infections of the mouth and throat (tonsillitis, laryngitis, aphthous ulcers, etc.). Strepsils are packed in vacuum-sealed tins of 24 individually-wrapped lozenges. Basic N.H.S. price Is. $9 \frac{1}{2} \mathrm{~d}$. per tin.

\section{'NIVEMYCIN' PREPARATIONS}

Boots Pure Drug Company Limited announce that the trade name ' Nivemycin' has been applied to their range of neomycin preparations. Neomycin Ointment-Boots, and Neomycin Eye Ointment-Boots, are, therefore, now available as 'Nivemycin' Ointment and 'Nivemycin' Eye Ointment; there are no changes in the formulae.

In addition, two new products have been added to the range: 'Nivemycin' Tablets (each tablet containing $0.5 \mathrm{~g}$. of neomycin sulphate, B.P.; 요 supplied in bottles of 20 at a retail price of $97 \mathrm{~s} .6 \mathrm{~d}$.) and 'Nivemycin' Lotion (an effective non-greasy $\mathcal{O}$ preparation containing 0.5 per cent. of neomycin $\omega$ sulphate, B.P.; the I-fl. oz. plastic applicatorbottle is available at a retail price of $14 \mathrm{~s}$.). These $\stackrel{\varrho}{\subset}$ additions enable a suitable 'Nivemycin' pre- $\mathbb{D}_{\mathscr{D}}$ paration to be selected for every therapeutic : need. 